Published in final edited form as:

Arch Biochem Biophys. 2016 February 15; 592: 27-37. doi:10.1016/j.abb.2016.01.009.

\title{
Functional importance of alle-346 and alle-348 in the catalytic sites of Escherichia coli ATP synthase
}

\author{
Chao Zhao ${ }^{1, \#}$, Hiba Syed ${ }^{1}$, Sherif S. Hassan ${ }^{2,4}$, Vineet K. Singh ${ }^{3}$, and Zulfiqar Ahmad ${ }^{1,}{ }^{*}$ \\ ${ }^{1}$ Department of Biochemistry, Kirksville College of Osteopathic Medicine, A.T. Still University, \\ Kirksville, Missouri 63501, USA \\ ${ }^{2}$ Department of Anatomy, Kirksville College of Osteopathic Medicine, A.T. Still University, \\ Kirksville, Missouri 63501, USA \\ ${ }^{3}$ Department of Microbiology \& Immunology, Kirksville College of Osteopathic Medicine, A.T. Still \\ University, Kirksville, Missouri 63501, USA \\ ${ }^{4}$ Faculty of Medicine, Cairo University, Cairo, Egypt
}

\begin{abstract}
We studied the functional role of highly conserved VISIT-DG sequence residues alle-346 and alle-348 in the catalytic sites of Escherichia coli $\mathrm{F}_{1} \mathrm{~F}_{\mathrm{o}}$ ATP synthase. alle-346 is in close proximity, 2.98 and $3.63 \AA$, to the two known phosphate binding residues aR376 and $\beta R 182$; alle-348 is situated within $3.66 \AA$ from $\beta R 182$. Single or double mutants of both aI346 and aI348 resulted in a variable loss of oxidative phosphorylation and ATPase activity. Azide, fluoroaluminate, and fluoroscandium caused insignificant to significant inhibition of mutants. Whereas the wild-type enzyme was completely inhibited by NBD-Cl (7-chloro-4-nitrobenzo-2oxa-1, 3-diazole), a variable extent of inhibition was observed for aI346 and aI348 mutants. MgPi protection against NBD-Cl induced inhibition of wild-type, aI346, and aI348 demonstrated that, although strongly conserved, $\alpha \mathrm{I} 346$ and aI 348 have no direct role in phosphate binding. Insertion

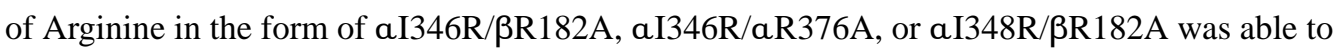
compensate for the absence of known phosphate-binding Arginine residues $\beta R 182$ and aR376. Results also suggest that alle-346 and alle-348 seem to have functional importance in upholding the phosphate-binding subdomain and transition state stabilization in the catalytic sites of E. coli ATP synthase.
\end{abstract}

Keywords

\footnotetext{
"Corresponding author: Department of Biochemistry, Kirksville College of Osteopathic Medicine, A.T. Still University, Kirksville, Missouri 63501; Phone: (660) 626-2144; Fax: (660) 626-2085; zahmad@ atsu.edu.

\#Currently at: Department of Biology, Georgia State University, Atlanta, Georgia 30303, USA

Publisher's Disclaimer: This is a PDF file of an unedited manuscript that has been accepted for publication. As a service to our customers we are providing this early version of the manuscript. The manuscript will undergo copyediting, typesetting, and review of the resulting proof before it is published in its final citable form. Please note that during the production process errors may be discovered which could affect the content, and all legal disclaimers that apply to the journal pertain.
} 


\section{Keywords}

$\mathrm{F}_{1} \mathrm{~F}_{\mathrm{o}}$ ATP synthase; E. coli ATP synthase; ATPase inhibition; oxidative phosphorylation; $\mathrm{Pi}$ binding residues

\section{Introduction}

$\mathrm{F}_{1} \mathrm{~F}_{\mathrm{o}}$ ATP synthase, the smallest known biological nanomotor, is the main source of ATP generation in almost all organisms. Structurally, Escherichia coli has the simplest form of ATP synthase with five subunits arranged in the water-soluble $F_{1}$ sector $\left(a_{3} \beta_{3} \gamma \delta \varepsilon\right)$ and three subunits in the membrane-associated $\mathrm{F}_{\mathrm{o}}$ sector $\left(\mathrm{ab}_{2} \mathrm{c}_{10}\right)$ [1, 2]. Structural and functional aspects of membrane bound $\mathrm{F}_{1} \mathrm{~F}_{\mathrm{o}}$ ATP synthase enzyme are highly conserved among different species. ATP hydrolysis and synthesis take place at the $\alpha / \beta$ interface, whereas proton transport occurs through the membrane-embedded $\mathrm{F}_{\mathrm{o}}$ sector $[3,4]$.

Catalytic and motor functions of ATP synthase are connected to the Pi (inorganic phosphate) binding residues and to residues surrounding the Pi binding subdomain in the catalytic sites [5]. MgPi protection against 7-chloro-4-nitrobenzo-2-oxa-1, 3,-diazole (NBD-Cl) induced inhibition of ATPase activity provides an indirect but useful method to measure Pi binding [6]. Because NBD-Cl covalently interacts with $\beta 297$ in the $\beta E$ catalytic site [7], the protection by Pi specifies that Pi binds at the $\beta E$ (empty) catalytic site. Modification of the Pi protection assay initially used in mitochondrial membranes for E. coli, purified $\mathrm{F}_{1}$ or membrane bound $\mathrm{F}_{1} \mathrm{~F}_{\mathrm{o}}$, allowed us to previously investigate the role of nine residues, $\beta$ Arg-246, $\beta$ Asn-243, a Arg-376, $\beta$ Lys-155, $\beta$ Arg-182, aPhe-291, aSer-347, Thr-349, and a Gly-351 ${ }^{1}$, in Pi binding. Four residues, $\beta$ Arg-246, a Arg-376, $\beta$ Lys-155, and $\beta$ Arg-182, were directly involved in Pi binding by forming a triangle across $\mathrm{Pi}$, and two residues, aSer-347 and a Thr-349, were indirectly involved in Pi binding through their interaction with $\beta$ Arg-246 and $\beta$ Arg-182 (see Fig. 1) [5, 8-15]. Three residues, $\beta$ Asn-243, $\alpha$ Phe-291, and aGly-351, did not have a direct role in Pi binding even though all the above residues are positioned in close proximity to the phosphate analogs $\mathrm{AlF}_{3}$ or $\mathrm{SO}_{4}{ }^{2-}$ in X-ray structures of catalytic sites. [16, 17]. Fig.1A shows an $\mathrm{SO}_{4}{ }^{2-}$ ion thought to mimic phosphate while in Fig.1B fluoroaluminate is in the position of the phosphate. aSer-347, a Thr-349, and a Gly-351 are all part of the highly conserved VISIT-DG sequence. The VISIT-DG sequence is situated in close proximity to the $\alpha / \beta$ interface flanking the Pi binding subdomain in the catalytic sites (Fig. 1B).

Menz et al [17] determined the bovine ATP synthase X-ray crystallographic structure that showed the transition state analog MgADP.AlF4- trapped in catalytic sites (Fig. 1B). In this complex, the fluoroaluminate group occupies the position of the ATP- $\gamma$-phosphate in the predicted transition state. Senior et al [3] proposed the mechanism of Pi and MgADP condensation. Pedersen's group [18] reported the transition state-like structure of $F_{1}$ from rat liver crystallized with the $\mathrm{Pi}$ analog vanadate $\left(\mathrm{V}_{\mathrm{i}}\right)$. The $\mathrm{MgV}_{\mathrm{i}}-\mathrm{F}_{1}$ complex inhibited the catalytic activity to the same extent as the MgADP-Vi- $\mathrm{F}_{1}$ complex, suggesting that ADP was

${ }^{1}$ E. coli residue numbers used throughout. 
not essential. In another study, we observed that neither purified $\mathrm{F}_{1}$ nor membrane bound $\mathrm{F}_{1} \mathrm{~F}_{\mathrm{o}}$ from $E$. coli was inhibited by MgVi or MgADP-Vi [9]. Therefore, fluoroaluminate and fluoroscandium were used to assess the potential to stabilize the transition state complex [5, $8-11,14,15]$.

The first high-resolution crystal structure of $E$. coli $\mathrm{F}_{1}$ sector was resolved by Cingolani and Duncan [19]. Resolution of the E. coli structure provides more opportunities for the development of effective antimicrobial drugs in comparison to mitochondrial ATP synthase $[19,20]$, such as the newly developed anti-tuberculosis drug bedaquiline that targets bacterial ATP synthase [21]. Unfortunately, the E. coli crystallographic structure [20] does not contain sulfate, phosphate, fluoroaluminate, fluoroscandium, or bound NBD-Cl making it unsuitable for the identification, characterization, and molecular modeling of Pi binding residues in the catalytic sites of E. coli. However, the mitochondrial ATP synthase structure with bound phosphate analogs and about $70 \%$ homology to the $E$. coli sequence can be used to study the Pi binding residues $[16,17,22]$.

The well-preserved nature and contiguous location of VISIT-DG sequence residues with the Pi binding subdomain in the catalytic sites is indicative of their direct or indirect role in Pi binding and maintenance of the Pi binding subdomain. In the absence of direct Pi binding or release assay $\mathrm{MgPi}$ protection against $\mathrm{NBD}-\mathrm{Cl}$ inhibition and the transition state analog induced inhibition profiles confidently allow us to investigate the direct or indirect role of residues in Pi binding. This manuscript describes the functional importance of two highly conserved a-subunit VISIT-DG sequence residues, alle-346 and alle-348, in Pi binding and their possibility of compensating for the other known Pi binding residues. Figure 1B shows the position of a Ile-346 and alle-348 residues with respect to other known Pi binding residues in the catalytic sites. The strongly conserved nature and proximity of aI346 and aI348 to the Pi binding subdomain residues resulted in the following study questions: What is the purpose of non-polar hydrophobic side chains of aI346 and aI348 in the polar Pi binding subdomain? Do alle-346 and alle-348 directly or indirectly participate in Pi binding? Will the mutations a $346 \mathrm{~A} / \mathrm{D} / \mathrm{Q} / \mathrm{R}$ or $\mathrm{aI} 348 \mathrm{~A} / \mathrm{D} / \mathrm{Q} / \mathrm{R}$ have any effect on transition state formation? Moreover, will aI346R or aI348R be able to compensate for the nearby known Pi binding residues $\beta$ Arg-246, $\beta$ Arg-182, or a Arg-376?

\section{Materials and Methods}

\section{E.coli wild-type and mutant strains}

The wild-type E. coli strain used was pBWU13.4/DK8 [23]. Mutant strains were generated by the Vandeyar et al method [24]. Oligonucleotide-directed mutagenesis was performed using the M13mp18 template containing the HindIII-Xba1 fragment from pSN6. Plasmid pSN6 expresses all the ATP synthase genes and has the $\beta$ Y $331 \mathrm{~W}$ mutation from plasmid pSWM4 [25] introduced on a Sac1-Eag1 fragment into pBWU13.4 [23]. Mutagenic oligonucleotides used in this study are listed in Table 1. The presence of correct mutations and absence of undesired changes in sequence was confirmed by DNA sequencing. All the mutations were transferred to pSN6 on a Csp451 (an isoschizomer of BstBI) and Pml1 fragment, generating the new single plasmids pZA65(aI346A), pZA66(aI346D), pZA67(aI346Q), pZA68(aI346R), pZA69(aI348A), pZA70(aI348D), pZA71(aI348Q), 
pZA72(aI348R), and pZA73(aR376A). Double mutants pZA74(aI346R/aR182A), pZA75(aI346R/aR376A), pZA76(aI346R/ßR246A), pZA77(aI348R/aR182A), pZA78 (aI348R/aR376A), and pZA79 (aI348R/3R246A) were generated by combining pZA65pZA72 fragments on pZA24, pZA73, and pZA7 plasmids. All plasmids were transformed into the DK8 strain [26] from which ATP synthase genes were deleted for expression of the mutant enzymes. All mutant strains also contained the $\beta Y 331 \mathrm{~W}$ mutation. This mutation is valuable tool for measuring nucleotide binding parameters [25] and has no significant effect on enzyme function. Although Trp mutation was not used in this study, the $\beta Y 331 \mathrm{~W}$ mutation was included for possible future use.

\section{Preparation of E. coli membranes, measurement of growth yield in limiting glucose medium, and assay of membrane bound $F_{1} F_{0}$ ATPase activity}

E. coli membrane bound $\mathrm{F}_{1} \mathrm{~F}_{\mathrm{o}}$ ATP synthase were prepared by the Senior et al method [27]. Notably in this procedure, initial membrane bound $\mathrm{F}_{1} \mathrm{~F}_{\mathrm{o}}$ pellets are washed three times. The first wash is done in a buffer containing $50 \mathrm{mM}$ TES pH 7.0, 15\% glycerol, $40 \mathrm{mM}$ 6aminohexanoic acid, and $5 \mathrm{mM}$ p-aminobenzamidine. The next two washes are carried out in a buffer containing $5 \mathrm{mM}$ TES pH 7.0, 15\% glycerol, $40 \mathrm{mM}$ 6-aminohexanoic acid, 5 $\mathrm{mM}$ p-aminobenzamidine, $0.5 \mathrm{mM}$ DTT, and $0.5 \mathrm{mM}$ EDTA. Before the experiments, membranes are washed two additional times by resuspension and ultracentrifugation in 50 $\mathrm{mM}_{\text {TrisSO}} \mathrm{pH}_{4} .0$ and $2.5 \mathrm{mM} \mathrm{MgSO}_{4}$. These extra washes are meant to reduce the null mutant to a true zero activity. Thus, low activities observed for mutants are their own and not that of any contaminants. Growth yield in limiting glucose was measured as described previously [28]. ATPase activity was measured in a $1 \mathrm{ml}$ ATP assay buffer containing 10

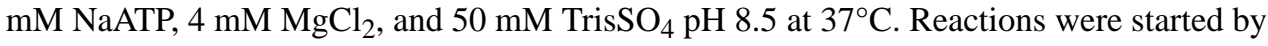
addition of membrane bound $\mathrm{F}_{1} \mathrm{~F}_{\mathrm{o}}$ and stopped by addition of $1 \mathrm{ml}$ SDS to a final concentration of 3.3\%. Taussky and Shorr reagent was used to measure the released Pi [29]. Reaction times for 20-30 $\mu \mathrm{g}$ wild-type membrane bound $\mathrm{F}_{1} \mathrm{~F}_{\mathrm{o}}$ protein were 5-10 min, and reaction times for $40-60 \mu \mathrm{g}$ membrane bound $\mathrm{F}_{1} \mathrm{~F}_{\mathrm{o}}$ protein were $30-50 \mathrm{~min}$. All reactions were found to be linear with respect to time and protein concentration. The purity of membrane bound $\mathrm{F}_{1} \mathrm{~F}_{\mathrm{o}}$ was checked by SDS-gel electrophoresis on $10 \%$ acrylamide gels and by immunoblotting with rabbit polyclonal anti- $\mathrm{F}_{1}-\mathrm{a}$ and anti- $\mathrm{F}_{1}-\beta$ antibodies as described previously [30] and shown in Fig 2.

\section{Inhibition of ATPase activity by NBD-Cl and protection by MgADP or MgPi}

NBD-Cl stock solution was prepared in dimethyl sulfoxide (DMSO) and protected from light. Membrane bound $\mathrm{F}_{1} \mathrm{~F}_{\mathrm{o}}(0.2-0.5 \mathrm{mg} / \mathrm{ml})$ were reacted with $\mathrm{NBD}-\mathrm{Cl}$ for $60 \mathrm{~min}$ in the dark, in T8 buffer (50 mM TrisSO 4 pH 8.0), containing $2.5 \mathrm{mM} \mathrm{MgSO}_{4}$ at room temperature then $50 \mu \mathrm{l}$ aliquots were transferred to $1 \mathrm{ml}$ of ATP assay buffer to determine ATPase activity. For protection from NBD-Cl inhibition, $\mathrm{F}_{1} \mathrm{~F}_{\mathrm{o}}$ membranes were preincubated 60 min with $\mathrm{ADP}$ or Pi at room temperature before addition of $\mathrm{NBD}-\mathrm{Cl} . \mathrm{MgSO}_{4}$ and $\mathrm{ADP}$ or Pi were present in equimolar concentrations in the reaction assay. Blank controls contained protein in T8 buffer with no added NBD-Cl. Up to $10 \mathrm{mM}$ of MgADP or $50 \mathrm{mM}$ of MgPi alone were shown to have no inhibitory effect. $[9,15]$. 


\section{Reversal of NBD-Cl inhibited ATPase activity by DTT}

For DTT induced reversal of NBD-Cl inhibition, membrane bound $\mathrm{F}_{1} \mathrm{~F}_{\mathrm{o}}$ were first reacted with NBD-Cl $(150 \mu \mathrm{M})$ for $1 \mathrm{~h}$ at room temperature in the dark. Then, $4 \mathrm{mM}$ DTT was added and incubation continued for $1 \mathrm{~h}$ at room temperature before ATPase assay. Control samples without NBD-Cl or DTT were included and incubated for the same time periods.

\section{Inhibition of ATPase activity by azide, fluoroaluminate, or fluoroscandium}

Azide induced inhibition was measured by preincubating membrane bound $\mathrm{F}_{1} \mathrm{~F}_{\mathrm{O}}$ with varied concentrations of sodium azide for $30 \mathrm{~min}$. Then, $1 \mathrm{ml}$ ATPase assay buffer was added to measure the activity. Fluoroaluminate or fluoroscandium induced inhibition was measured by incubating membrane bound $\mathrm{F}_{1} \mathrm{~F}_{\mathrm{o}}$ for $60 \mathrm{~min}$ at room temperature in $50 \mathrm{mM}^{\text {TrisSO }} \mathrm{S}_{4}, 2.5$ $\mathrm{mM} \mathrm{MgSO}_{4}, 1 \mathrm{mM} \mathrm{NaADP}$, and $10 \mathrm{mM} \mathrm{NaF}$ at a protein concentration of $0.2-0.5 \mathrm{mg} / \mathrm{ml}$ in the presence of varied concentrations of $\mathrm{AlCl}_{3}$ or $\mathrm{ScCl}_{3}$. A $1 \mathrm{ml}$ ATPase assay buffer was then added to $50 \mu \mathrm{l}$ reaction samples to measure the ATPase activity. Omission of $\mathrm{MgSO}_{4}$, $\mathrm{NaADP}$, or NaF resulted in no inhibition of membranes.

\section{Results}

\section{Growth properties of single and double mutants of E. coli ATP synthase}

Nine new single mutants, aI346A, aI346D, aI346Q, aI346R, aI348A, aI348D, aI348Q, aI348R, and aR376A, and six double mutants, $\alpha \mathrm{I} 346 \mathrm{R} / \beta \mathrm{R} 182 \mathrm{~A}, \mathrm{aI} 346 \mathrm{R} / \beta \mathrm{R} 246 \mathrm{~A}$,

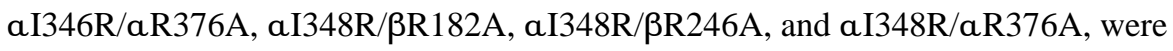
generated. Despite non-polar hydrophobic side chains, alle-346 and alle-348 are strongly conserved in the aVISIT-DG sequence and are also in close proximity to positively charged Pi binding residues in the catalytic sites of ATP synthase. aI346A and aI348A mutants were constructed to appreciate the role of non-polar hydrophobic side chains in Pi binding and the transition state. a I346Q and aI348Q mutants were designed to investigate the impact of the larger side chain of Gln on aI346 and aI348. aI346D, aI348D, aI346R, and aI348R were generated to determine the impact of negative and positive charges on the nearby known Pi binding residues $\beta$ R246, $\beta R 182$, and $\alpha$ R376. Growth properties of $\beta R 246 A, \beta R 182 A$, and aR376A were in excellent agreement with previously published data $[3,9,12,15,31]$. Our

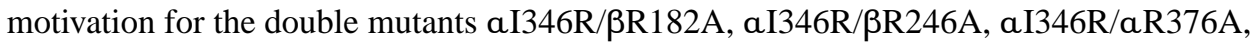

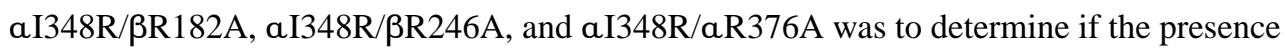
of the Arg side chain on aI346R or aI348R could compensate for absence of the Arg side chain on $\beta$ R182A or aR376A.

Table 2 shows that the introduction of Ala, Asp, Gln, or Arg as aI346A, aI346D, aI346Q, aI346R, aI348A, aI348D, aI348Q, and aI348R caused some loss of oxidative phosphorylation as represented by loss of growth on succinate and limiting glucose mediums. Replacement of Arg into Ala in three known Pi binding residues $\beta$ R182A, $\beta R 246 \mathrm{~A}$, and aR376A resulted in significant loss of oxidative phosphorylation and reduction in ATPase activity. Considerable oxidative phosphorylation was retained by the

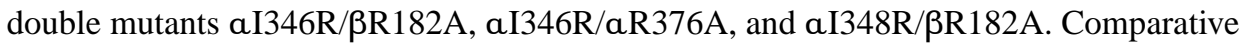
specific ATPase activity values of membrane bound $\mathrm{F}_{1} \mathrm{~F}_{\mathrm{o}}$ preparations containing wild-type, null, and mutant enzymes are also shown in Table 2. Variable reduction in ATPase activity 
was observed for all mutant enzymes. Double mutants aI346R/3R182A, aI346R/aR376A, and $\mathrm{\alpha I} 348 \mathrm{R} / \beta \mathrm{R} 182 \mathrm{~A}$ augmented the ATPase activity by about 70 -fold from $0.07,0.065$ to $4.61,4.97$, and 4.87, respectively. Purity and integrity of $\alpha$ and $\beta$ subunits in wild-type and mutant membrane bound $\mathrm{F}_{1} \mathrm{~F}_{\mathrm{o}}$ were in excellent agreement with previously published data $[8,30]$. This result also confirmed that the reduced ATPase in mutants was not due to contaminants, impaired assembly of ATP synthase, or loss of $\mathrm{F}_{1}$ during membrane preparation, thus attributing the low mutant activities to mutant $\mathrm{F}_{1}$.

\section{Inhibition of membrane bound $F_{1} F_{0}$ ATPase activity by NBD-Cl and reversal by DTT}

In previous studies, both membrane bound $\mathrm{F}_{1} \mathrm{~F}_{\mathrm{o}}$ and purified $\mathrm{F}_{1}$ preparations provided equivalent assay results $[5,8,9,14,15,30,32-35]$. Therefore, all the inhibition assays in this study were carried out using membrane bound $\mathrm{F}_{1} \mathrm{~F}_{\mathrm{o}}$ because it is convenient and less time consuming. Figure 3 shows NBD-Cl induced inhibition of wild-type and mutant membranes in the presence of varied concentrations of NBD-Cl. NBD-Cl resulted in complete inhibition of wild-type with no residual activity, which was consistent with previous studies [5, 8-12, 14, 15]. aI346A, aI346Q, aI346R, aI348A, aI348Q, aI346R/ $\beta R 182 \mathrm{~A}, \alpha \mathrm{I} 346 \mathrm{R} / \mathrm{\alpha R} 376 \mathrm{~A}$, and $\mathrm{\alpha I} 348 \mathrm{R} / \beta \mathrm{R} 182 \mathrm{~A}$ mutants were also almost completely inhibited. Variable degrees of inhibition were observed for other single or double mutants at about $59 \%$ for a I346D, 34\% for a I348D, $40 \%$ for a I348R, $61 \%$ for aR $376 \mathrm{~A}, 54 \%$ for

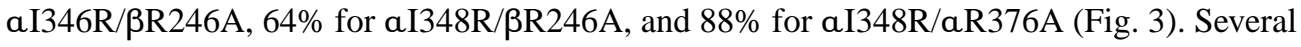
examples of incomplete or partial inhibition of mutant enzymes by NBD-Cl are documented in previous studies $[5,8-12,14,15]$. To validate that the maximal reaction with NBD-Cl had been reached, partially inhibited membrane bound $\mathrm{F}_{1} \mathrm{~F}_{\mathrm{O}}$ mutant enzymes were incubated with $150 \mu \mathrm{M} \mathrm{NBD}-\mathrm{Cl}$ for $1 \mathrm{~h}$ as in Figure 3, followed by an additional amount of $200 \mu \mathrm{M}$ NBD-Cl (totaling $350 \mu \mathrm{M}$ ) and continuing the incubation for an extra hour before measuring ATPase activity. No significant change in inhibition confirmed that the reaction of NBD-Cl was complete and that fully reacted aI346D, aI348D, aI348R, aR376A, aI346R//R182A, aI348R/ $\beta R 246$, and $\alpha \mathrm{I} 348 \mathrm{R} / \mathrm{aR} 376 \mathrm{~A}$ membrane bound $\mathrm{F}_{1} \mathrm{~F}_{\mathrm{o}}$ mutants retained residual activity (Fig. 4A). We also determined if inhibition by NBD-Cl could be reversed by the addition of the reducing agent DTT since reversibility by DTT has been shown to be indicative of reaction specificity at $\beta$ Try-297 [36, 37]. For this experiment, DTT restored near normal ATPase activity in all cases (Fig. 4B), verifying that NBD-Cl reacts specifically with residue $\beta$ Tyr-297 in the wild-type and in mutants.

\section{Protection against NBD-Cl inhibition of ATPase activity by MgADP or MgPi}

Figure 5 shows the $\mathrm{MgADP}$ protection data against $\mathrm{NBD}-\mathrm{Cl}$ in wild-type and mutant membrane bound $\mathrm{F}_{1} \mathrm{~F}_{\mathrm{o}}$ enzymes. Previous research has shown that protection of NBD-Clinduced inhibition of wild-type and a variety of mutant membrane bound $\mathrm{F}_{1} \mathrm{~F}_{\mathrm{o}}$ or water soluble $\mathrm{F}_{1}$ occurs at high concentrations of MgADP. In this study too, high concentrations of MgADP were required to protect wild-type and mutant enzymes against NBD-Cl inhibition. High MgADP concentrations efficiently keep the $\beta E$ site occupied in time average and thus hold back the access to NBD-Cl by sterically obstructing the site [5, 8-15]. Therefore, these results prove that $\mathrm{NBD}-\mathrm{Cl}$ is reacting in $\beta \mathrm{E}$ in the mutants and that the ATPase activities measured in the mutants are actually of ATP synthase enzyme and not due to any 
contaminant. This scheme is consistent with the X-ray crystallographic studies of Orris et al concluding NBD-Cl reacts specifically in the $\beta E$ catalytic site [7].

$\mathrm{MgPi}$ protection against NBD-Cl reaction is shown in Figure 6. Pi protected fully against NBD-Cl inhibition of ATPase activity in wild-type and in aI346A, aI346D, aI346Q, aI346R, aI348A, aI348D, aI348Q, aI348R, aI346R/ßR182A, a 346R/aR376A, and aI348R/ $\beta R 182 \mathrm{~A}$ mutants. Pi did not protect against NBD-Cl inactivation in aI346R/ $\beta R 246 \mathrm{~A}, a I 348 \mathrm{R} / \beta \mathrm{R} 246 \mathrm{~A}$, or aI348R/aR376A mutants.

\section{Inhibition of ATPase activity by fluoroaluminate, fluoroscandium, and azide}

Effects of transition and ground state analogs were studied. Figures 7A and 7B show the inhibition of wild-type and mutant enzymes by fluoroaluminate and fluoroscandium, respectively. $\mathrm{AlCl}_{3}$ induced complete inhibition of wild-type, a I346A, a I346Q, and aI348A. Maximal inhibition by $\mathrm{AlCl}_{3}$ for other mutants was observed at about $17 \%$ for aI346D, 84\% for aI346R, 23\% for a I348D, 86\% for a I348Q, 0\% for aI348R, 93\% for

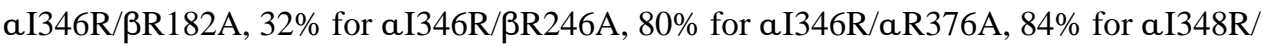
$\beta R 182 \mathrm{~A}, 20 \%$ for $\mathrm{aI} 348 \mathrm{R} / \beta \mathrm{R} 246 \mathrm{~A}$, and $42 \%$ for $\mathrm{aI} 348 \mathrm{R} / \mathrm{aR} 376 \mathrm{~A} . \mathrm{ScCl}_{3}$ induced nearly complete inhibition of wild-type. $\mathrm{ScCl}_{3}$ caused variable degrees of inhibition at about $66 \%$ for aI346A, $0 \%$ for a I346D, 80\% for a I346Q, 15\% for a 3466R, 84\% for aI348A, 30\% for aI348D, 65\% for aI348Q, 30\% for aI348R, 80\% for aI346R/ $\beta \mathrm{R} 182 \mathrm{~A}, 6 \%$ for aI346R/

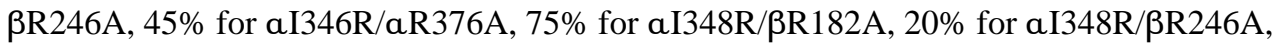
and $30 \%$ for aI348R/aR376A in mutants. Sodium azide is a known potent inhibitor of ATP synthase. As seen in Figure 8, azide strongly inhibited wild-type enzyme and caused varied degrees of inhibition in mutants at about $44 \%$ for a I346A, 20\% for aI346D, 20\% for aI346Q, 43\% for aI346R, 29\% for a I348A, 0\% for aI348D, 0\% for aI348Q, 0 for aI348R,

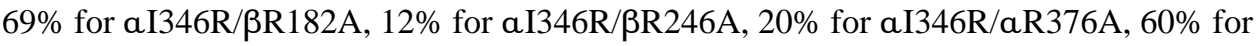

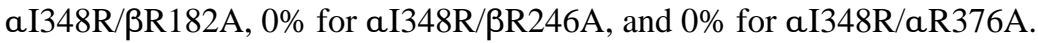

\section{Discussion}

The purpose of this study was to understand the functional importance of non-polar hydrophobic side chains of alle-346 and alle-348 residues in the highly conserved aVISITDG sequence of E. coli ATP synthase. All the VISIT-DG sequence residues are located in close proximity to the positively charged Pi binding residues in the catalytic sites. aI346 is only 2.98 and $3.63 \AA$ from aR376 and $\beta$ R182, two known Pi binding residues. $\beta R 246$, another known Pi binding residue, is $7.41 \AA$ apart from aI346. The distance between aI348 and $\beta$ R182 is only $3.66 \AA$, but aR376 and $\beta$ R246 are located at 5.29 and $5.77 \AA$ (Fig. 1B). The side chains of aI346 and a I348 are also in close proximity to the bound Pi analogs $\mathrm{AlF}_{3}, \mathrm{AlF}_{4}{ }^{-}$, and $\mathrm{SO}_{4}{ }^{2-}[16,17] . \mathrm{AlF}_{4}{ }^{-}$is located within 4.08 and $4.74 \AA$ from aI346 and aI348 (Fig. 1). Pi binding is a prerequisite for ATP synthesis by ATP synthase. Mutagenic analysis along with molecular modulations provides the opportunity to elucidate and understand the functional role of residues in Pi binding.

In previously published studies that used inhibitory effects of $\mathrm{Pi}$ analogs and the $\mathrm{MgPi}$ protection assay against NBD-Cl inhibition on mutant and wild-type ATP synthase, we demonstrated the functional role(s) of Pi binding subdomain and surrounding residues in Pi 
binding [5, 8-15]. Biochemical and biophysical characterization of nine catalytic site residues identified six residues, a Arg-376, $\beta$ Arg-182, $\beta$ Arg-246, $\beta$ Lys-155, aSer-347, and a Thr-349, directly or indirectly involved in Pi binding. While four residues, a Arg-376, $\beta$ Arg-182, $\beta$ Arg-246, and $\beta$ Lys-155, were directly involved in Pi binding, the two residues, aSer-347 and aThr-349, supported Pi binding and transition state stabilization through their interaction with $\beta$ Arg-246 and $\beta$ Arg-182 [5, 8-15]. These residues were shown to form a triangular subdomain around the $\mathrm{Pi}$ analogs $\mathrm{AlF}_{3}, \mathrm{AlF}_{4}{ }^{-}$, or $\mathrm{SO}_{4}{ }^{2-}$ within the catalytic site (Fig. 1B). Three other residues, $\beta$ N243, aF291, and aG351, although important for function were not involved in direct Pi binding. In previous studies, we also demonstrated that the introduction of a negative or positive charge in Pi binding subdomain resulted in strong variations of Pi binding $[8,10,14,15]$, indicating the presence of charged residues in the vicinity of the triangle was an important determinant of Pi binding. In this study, we used a similar approach by replacing alle-346 and alle-348 residues with a variety of residues Ala, Asp, Gln, or Arg.

Construction of aI346A, aI346D, aI346Q, aI346R, aI348A, aI348D, aI348Q, aI348R,

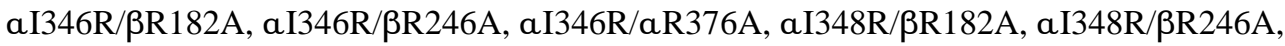
or aI348R/aR376A mutants did not affect assembly or structural integrity of the membrane bound ATP synthase. The content of $F_{1}-a$ and $F_{1}-\beta$ subunits in mutant enzymes was comparable to wild-type. As shown in Table 2, Mutations caused insignificant to substantial loss of oxidative phosphorylation and reduction in ATPase activity in mutant enzymes as suggested by growth on succinate or limiting glucose medium. A wide range of reduction in ATPase activity between 4-fold to 700-fold was observed for the mutant enzymes.

As shown in Figure 6, MgPi protected against NBD-Cl inhibition of all alle-346 and alle-348 single mutants, demonstrating Pi binding in the absence of aI346 or aI348. These results suggest that a-subunit VISIT-DG sequence residues a Ile-346 and alle-348 play no direct role in catalysis. While no Pi binding was observed in double mutants aI346R/

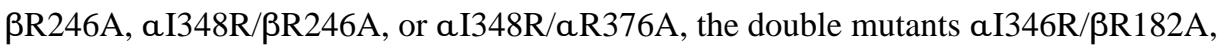
aI346R/aR376A, and aI348R/ $\beta$ R182A allowed Pi binding with substantial retention of oxidative phosphorylation and ATPase activity (Fig. 6). Clearly, insertion of Arg at alle-346 or alle-348 positions can compensate for the loss of Arg at $\beta$ Arg-182 or a Arg-376 but not at $\beta$ Arg-246.

MgADP-fluoroaluminate and MgADP-fluoroscandium are known potent inhibitors of wildtype E. coli ATP synthase, and both are believed to mimic the chemical transition state [5, $8-12,14,15,31,38]$. Transition state-like structures involving bound $\mathrm{MgADP}_{-} \mathrm{AlF}_{4}{ }^{-}$ complex have been seen in catalytic sites in ATP synthase by X-ray crystallography [17]. As shown in Figure 7, fluoroaluminate caused complete inhibition of wild-type, aI346A, aI346Q, and aI348A but resulted in variable degrees of inhibition for other mutants, indicating a partial to strong destabilization of the transition state. As expected, fluoroscandium also induced almost complete inhibition of wild-type enzyme but caused nearly complete to no inhibition of mutants, suggesting no destabilization to strong destabilization of the transition state. These results correspond with the amount of oxidative phosphorylation and ATPase activity found in each of the mutants. The higher degree of

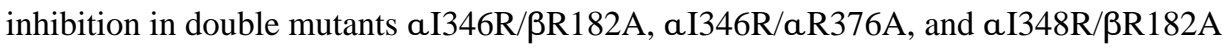


indicates some rescue of the transition state. Overall, these results suggest alle-346 and aIle-348 play a direct role in the transition state.

Azide induced X-ray crystallographic studies of ATP synthase [39] showed that azide inhibits ATP synthase by forming a tight-binding MgADP-azide complex in $\beta$ DP catalytic sites resembling MgADP-beryllium fluoride. Therefore, azide may be considered an analog of the MgATP ground-state. In the MgADP-azide complex, the azide position corresponds to MgATP gamma phosphate [39]. As shown in Figure 8, azide caused almost complete inhibition of wild-type membrane bound $\mathrm{F}_{1} \mathrm{~F}_{\mathrm{O}}$ and resulted in variable degrees of inhibition for mutants. Thus, all mutants seem to have some effect on substrate binding because of an effect at the gamma-P position. In single molecule experiments, Pi binding and release events have been directly linked to rotation of the central stalk [40]. Perturbation of the Pi binding site might disturb the integrity of the link between Pi binding and rotation and result in uncoupling. Therefore, alle-346 and alle-348 mutation data strongly suggest that both residues are required for transition state stabilization and not for Pi binding.

Because Arg residues occur frequently in catalytic sites in proteins (30), modulating the number of Arg residues in the Pi binding subdomain of ATP synthase is an advantageous approach to study their role in catalysis or Pi binding. Residues alle-346 and aI348 are 3.63 $\mathrm{a}$ and $3.66 \AA$, $7.41 \AA$ and $5.77 \AA$, $2.98 \AA$ and $5.29 \AA$ from known Pi binding residues $\beta A r g-182, \beta R 246$, and a Arg-376, respectively. Phosphate analogs $\mathrm{AlF}_{4}$ and $\mathrm{SO}_{4}{ }^{2-}$ are 4.08 $\AA$ and $5.65 \AA$ from aI346 and $4.74 \AA$ and $6.56 \AA$ from aI348. The distance between aI346 and aI 348 is $5.10 \AA$ (nearest atom distances quoted in all cases) using PDB 1H8E coordinates [17]. Thus, one of the experimental approaches we used was to introduce the mutation aI346R or aI348R in the wild-type background (with $\beta$ Arg-182, $\beta$ Arg-246, $\alpha$ Arg-376) and in the presence of the $\beta \mathrm{R} 182 \mathrm{~A}, \beta \mathrm{R} 246 \mathrm{~A}$, or aR376A mutation. The position of $\alpha \mathrm{I} 346$ and $\mathrm{aI} 348$ on top of the Pi binding pocket across the catalytic $\alpha / \beta$ interface with side-chains pointing toward the bound $\mathrm{Pi}$ analogs also makes a I346 and aI348 suitable candidates for the insertion of a new Arg. Introduction of Arg at aI346R or aI348R places extra positive charge fairly close to Pi. Further, double mutations aI346R/ $\beta R 182 A$, aI346R/

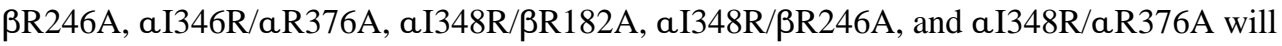
allow the Arg to fit into the large "hole" generated by $\beta$ Ala-182, $\beta$ Ala-246, or aAla-376 mutation. The $\beta$ R182A, $\beta$ R246A, or aR376A mutants did not allow Pi binding, but the a I346R or a I348R "rescued" Pi binding in combination with $\beta$ R182A or aR376A but not with $\beta$ R246A (Fig. 6). Previously [9] it was observed that introduction of Lys as a positive in place of Arg in the form of $\beta R 246 \mathrm{~K}$ could not compensate for the loss of Arg from the known Pi binding residue $\beta$ R246 therefore we have focused on introduction of Arg residue. Moreover, Lys in many other studies failed to compensate for the loss of Arg (Z. Ahmad unpublished data). Although, not checked in this study but it would be interesting to see if Lys can support the loss of Arg in phosphate binding subdomain.

Based on the loss of oxidative phosphorylation as manifested by growth on succinate or limiting glucose medium along with an almost 6-fold lower ATPase activity, a Arg-346 and a Arg-348 could be expected to assume the identical stereochemical interactions of $\beta A r g-182$ or a Arg- 376 . Electrostatic interactions are critical for the catalysis process, and we conclude that the presence of at least one positive charge at this general location is an 
essential determinant for the initiation of Pi binding in $\beta E$ catalytic site. In addition, extra positive charge in the form of aI346R or aI348R or extra negative charge in the form of aI346D or aI348D in wild-type background did not abrogate Pi binding (Fig. 6). Although, we previously found that the presence of negative charge in the form of aT349D prevented Pi binding by virtue of neutralizing the positive charge of nearby residue $\beta$ Arg-182 [15].

In summary, the highly conserved a-subunit VISIT-DG sequence residues alle-346 and alle-348 in E. coli ATP synthase seem to be required for function and transition state stabilization but appear to have no direct role in Pi binding. Introduction of Arg at these sites can compensate for the absence of Arg at known Pi binding residue sites $\beta$ Arg-182 or a Arg-376.

\title{
Acknowledgments
}

We are thankful to Deborah Goggin, scientific writer, research support, A.T. Still University for reviewing the manuscript. This work was supported by the National Institutes of Health, grant no. GM085771, and by an A.T. Still University Warner/Fermaturo Research Grant, grant no. 501-461, to ZA.

\author{
Abbreviations used \\ NBD-Cl 7-chloro-4-nitrobenzo-2-oxa-1,3-diazole \\ DTT dithiothreitol \\ EDTA Ethylenediaminetetraacetic acid \\ TES 2-[Tris(hydroxymethyl)methylamino]-1-ethanesulfonic acid \\ DMSO dimethyl sulfoxide
}

\section{References}

1. Senior AE. J Biol Chem. 2012; 287:30049-30062. [PubMed: 22822068]

2. Ahmad Z, Cox JL. The Scientific World Journal. 2014; 2014:10.

3. Senior AE, Nadanaciva S, Weber J. Biochim. Biophys. Acta. 2002; 1553:188-211. [PubMed: 11997128]

4. Abrahams JP, Leslie AGW, Lutter R, Walker JE. Nature. 1994; 370:621-628. [PubMed: 8065448]

5. Li W, Brudecki LE, Senior AE, Ahmad Z. J. Biol. Chem. 2009; 284:10747-10754. [PubMed: 19240022]

6. Perez JA, Greenfield AJ, Sutton R, Ferguson SJ. FEBS Lett. 1986; 198:113-118. [PubMed: 2869972]

7. Orriss GL, Leslie AG, Braig K, Walker JE. Structure. 1998; 6:831-837. [PubMed: 9687365]

8. Ahmad Z, Senior AE. J. Biol. Chem. 2005; 280:27981-27989. [PubMed: 15939739]

9. Ahmad Z, Senior AE. J. Biol. Chem. 2004; 279:31505-31513. [PubMed: 15150266]

10. Ahmad Z, Senior AE. J. Biol. Chem. 2004; 279:46057-46064. [PubMed: 15322126]

11. Ahmad Z, Senior AE. J. Bioenerg. Biomembr. 2005; 37:437-440. [PubMed: 16691479]

12. Ahmad Z, Senior AE. FEBS Lett. 2005; 579:523-528. [PubMed: 15642370]

13. Ahmad Z, Senior AE. FEBS Lett. 2006; 580:517-520. [PubMed: 16405964]

14. Brudecki LE, Grindstaff JJ, Ahmad Z. Arch. Biochem. Biophys. 2008; 471:168-175. [PubMed: 18242162]

15. Ahmad Z, Winjobi M, Kabir MA. Biochemistry. 2014; 53:7376-7385. [PubMed: 25375895] 
16. Braig K, Menz RI, Montgomery MG, Leslie AG, Walker JE. Structure. 2000; 8:567-573. [PubMed: 10873854]

17. Menz RI, Walker JE, Leslie AG. Cell. 2001; 106:331-341. [PubMed: 11509182]

18. Chen C, Saxena AK, Simcoke WN, Garboczi DN, Pedersen PL, Ko YH. J Biol Chem. 2006; 281:13777-13783. [PubMed: 16531409]

19. Cingolani G, Duncan TM. Nat Struct Mol Biol. 2011; 18:701-707. [PubMed: 21602818]

20. Roy A, Hutcheon ML, Duncan TM, Cingolani G. Acta Crystallogr Sect F Struct Biol Cryst Commun. 2012; 68:1229-1233.

21. Andries K, Verhasselt P, Guillemont J, Gohlmann HW, Neefs JM, Winkler H, Van Gestel J, Timmerman P, Zhu M, Lee E, Williams P, de Chaffoy D, Huitric E, Hoffner S, Cambau E, TruffotPernot C, Lounis N, Jarlier V. Science. 2005; 307:223-227. [PubMed: 15591164]

22. Walker JE, Saraste M, Runswick MJ, Gay NJ. EMBO J. 1982; 1:945-951. [PubMed: 6329717]

23. Ketchum CJ, Al-Shawi MK, Nakamoto RK. Biochem. J. 1998; 330:707-712. [PubMed: 9480879]

24. Vandeyar MA, Weiner MP, Hutton CJ, Batt CA. Gene. 1988; 65:129-133. [PubMed: 2840354]

25. Weber J, Wilke-Mounts S, Lee RS, Grell E, Senior AE. J. Biol. Chem. 1993; 268:20126-20133. [PubMed: 8376371]

26. Klionsky DJ, Brusilow WS, Simoni RD. J. Bacteriol. 1984; 160:1055-1060. [PubMed: 6238948]

27. Senior AE, Langman L, Cox GB, Gibson F. Biochem. J. 1983; 210:395-403. [PubMed: 6222731]

28. Senior AE, Latchney LR, Ferguson AM, Wise JG. Arch. Biochem. Biophys. 1984; 228:49-53. [PubMed: 6230049]

29. Taussky HH, Shorr E. J. Biol. Chem. 1953; 202:675-685. [PubMed: 13061491]

30. Ahmad Z, Laughlin TF, Kady IO. PLoS One. 2015; 10:e0127802. [PubMed: 25996607]

31. Nadanaciva S, Weber J, Senior AE. Biochemistry. 2000; 39:9583-9590. [PubMed: 10924155]

32. Chinnam N, Dadi PK, Sabri SA, Ahmad M, Kabir MA, Ahmad Z. Int. J. Biol. Macromol. 2010; 46:478-486. [PubMed: 20346967]

33. Dadi PK, Ahmad M, Ahmad Z. Int. J. Biol. Macromol. 2009; 45:72-79. [PubMed: 19375450]

34. Laughlin TF, Ahmad Z. Int. J. Biol. Macromol. 2010; 46:367-374. [PubMed: 20100509]

35. Ahmad Z, Ahmad M, Okafor F, Jones J, Abunameh A, Cheniya RP, Kady IO. Int. J. Biol. Macromol. 2012; 50:476-486. [PubMed: 22285988]

36. Ferguson SJ, Lloyd WJ, Radda GK. Eur. J. Biochem. 1975; 54:127-133. [PubMed: 238840]

37. Ferguson SJ, Lloyd WJ, Lyons MH, Radda GK. Eur. J. Biochem. 1975; 54:117-126. [PubMed: 238839]

38. Nadanaciva S, Weber J, Senior AE. J. Biol. Chem. 1999; 274:7052-7058. [PubMed: 10066761]

39. Bowler MW, Montgomery MG, Leslie AG, Walker JE. Proc Natl Acad Sci U S A. 2006; 103:8646-8649. [PubMed: 16728506]

40. Adachi K, Oiwa K, Nishizaka T, Furuike S, Noji H, Itoh H, Yoshida M, Kinosita K Jr. Cell. 2007; 130:309-321. [PubMed: 17662945] 


\section{Highlights}

- $\quad$ Role of highly conserved aI346 and aI348 VISIT-DG sequence residues in Pi binding is proposed

- $\quad$ Both aI346 and aI348 residues are required for upholding the phosphate binding sub-domain.

- $\quad$ Both aI346 and aI348 residues are required for the transition state stabilization.

- Introduction of Arg in place of Ile can compensate for the loss of an Arg involved in Pi binding 
(A)

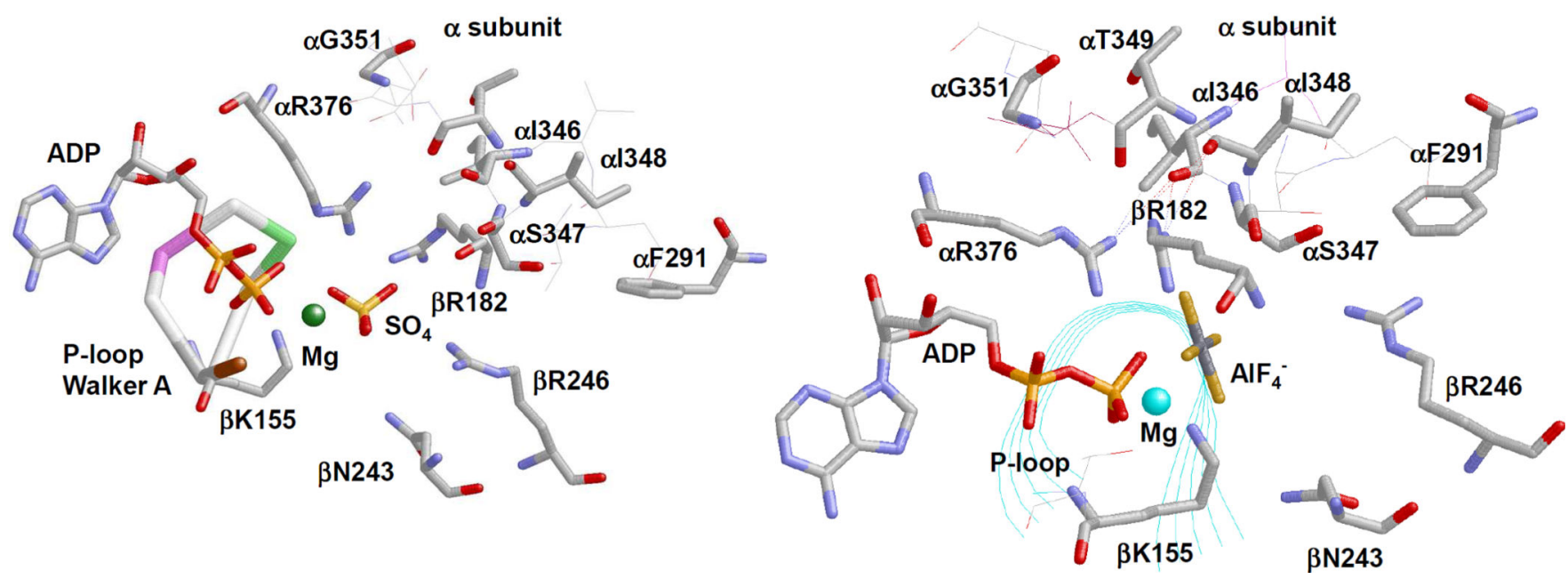

Figure 1. X-ray structures of ATP synthase catalytic sites showing spatial relationship of aVISIT-DG sequence residue $\alpha$ I346 and aI348

(A) The $\beta \mathrm{ADP}+\mathrm{Pi}\left(\beta\right.$-half-closed site) site in the $\mathrm{AlF}_{4}{ }^{-}$-inhibited enzyme [17]. The sulfate ion is thought to occupy the position of natural Pi. (B) The $\beta \mathrm{DP}$ site in the $\mathrm{AlF}_{4}{ }^{-}$-inhibited enzyme. [17]. Figure was generated by PDB files 1H8E using Rasmol software. Residue numbers are based on $E$. coli numbering. 


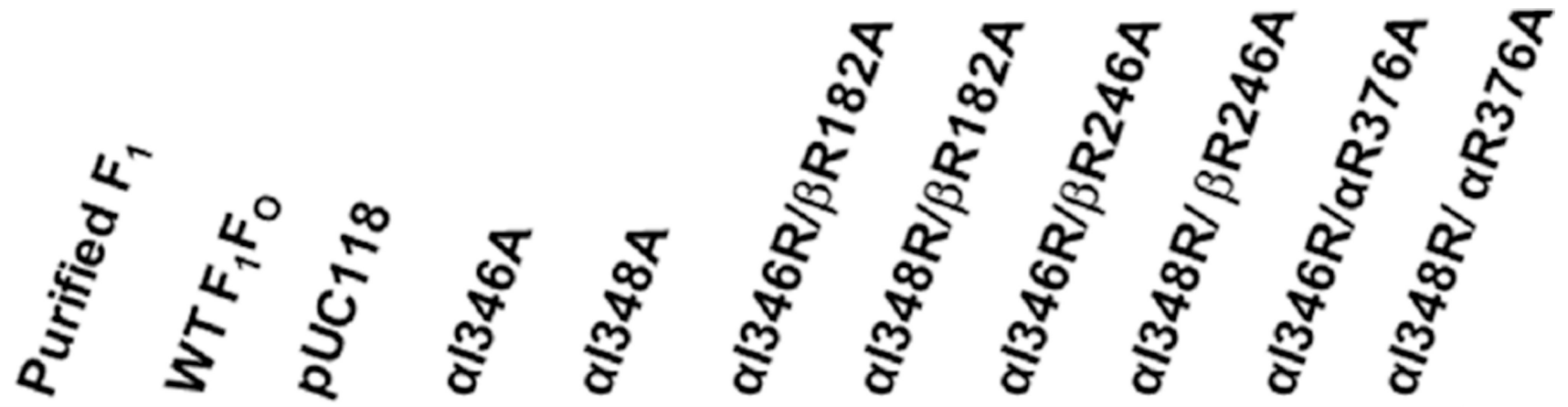

Figure 2. Immunoblot of wild-type purified $F_{1}$, wild-type membrane bound $F_{1} F_{0}$, and mutant membrane bound F1Fo ATP synthase with anti- $F_{1-a}$ antibody

Wild-type (WT) purified $\mathrm{F}_{1}(0.4 \mu \mathrm{g})$, WT membrane bound $\mathrm{F}_{1} \mathrm{~F}_{\mathrm{o}}$, and mutant membrane bound $\mathrm{F}_{1} \mathrm{~F}_{\mathrm{o}}$ preparations $(4 \mu \mathrm{g})$ were run on $10 \%$ SDS-polyacrylamide gel along with null mutant pUC118 $(4 \mu \mathrm{g})$. Protein bands were transferred to nitrocellulose and immunoblotted using anti- $\mathrm{F}_{1}-\mathrm{a}$ antibody. 

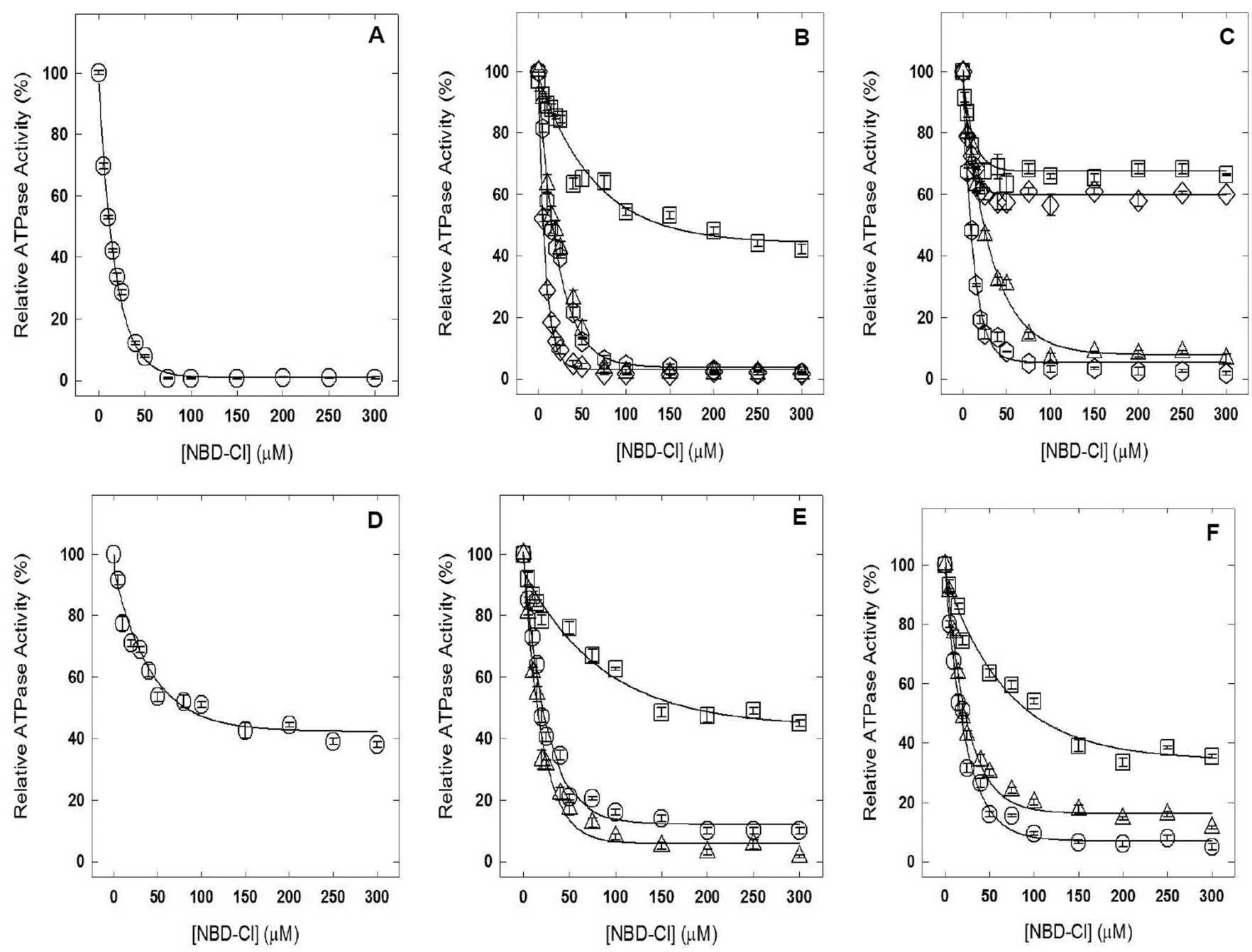

Figure 3. Inhibition of wild-type and mutant membrane bound $F_{1} F_{0}$ ATP synthase by NBD-Cl Membranes were preincubated for $60 \mathrm{~min}$ at room temperature with varied concentrations of NBD-Cl. $1 \mathrm{ml}$ ATP cocktail assay buffer was added to start the reaction. More details are provided in the Materials and Methods. A: wild-type (O); B: aI346A (๑), aI346D ( $\square$ ), aI346Q $(\Delta)$, and aI346R $(\diamond)$; C: aI348A $(\oslash)$, aI348D (口), aI348Q $(\Delta)$, and aI348R $(\diamond)$;

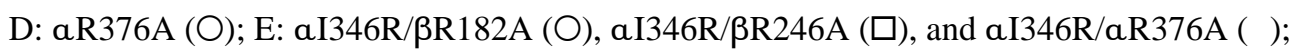

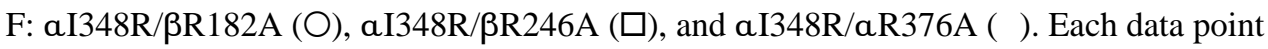
represents an average of at least three experiments with duplicate samples, using 2-3 independent membrane bound $\mathrm{F}_{1} \mathrm{~F}_{\mathrm{O}}$ preparations. Results agreed within $\pm 10 \%$. 

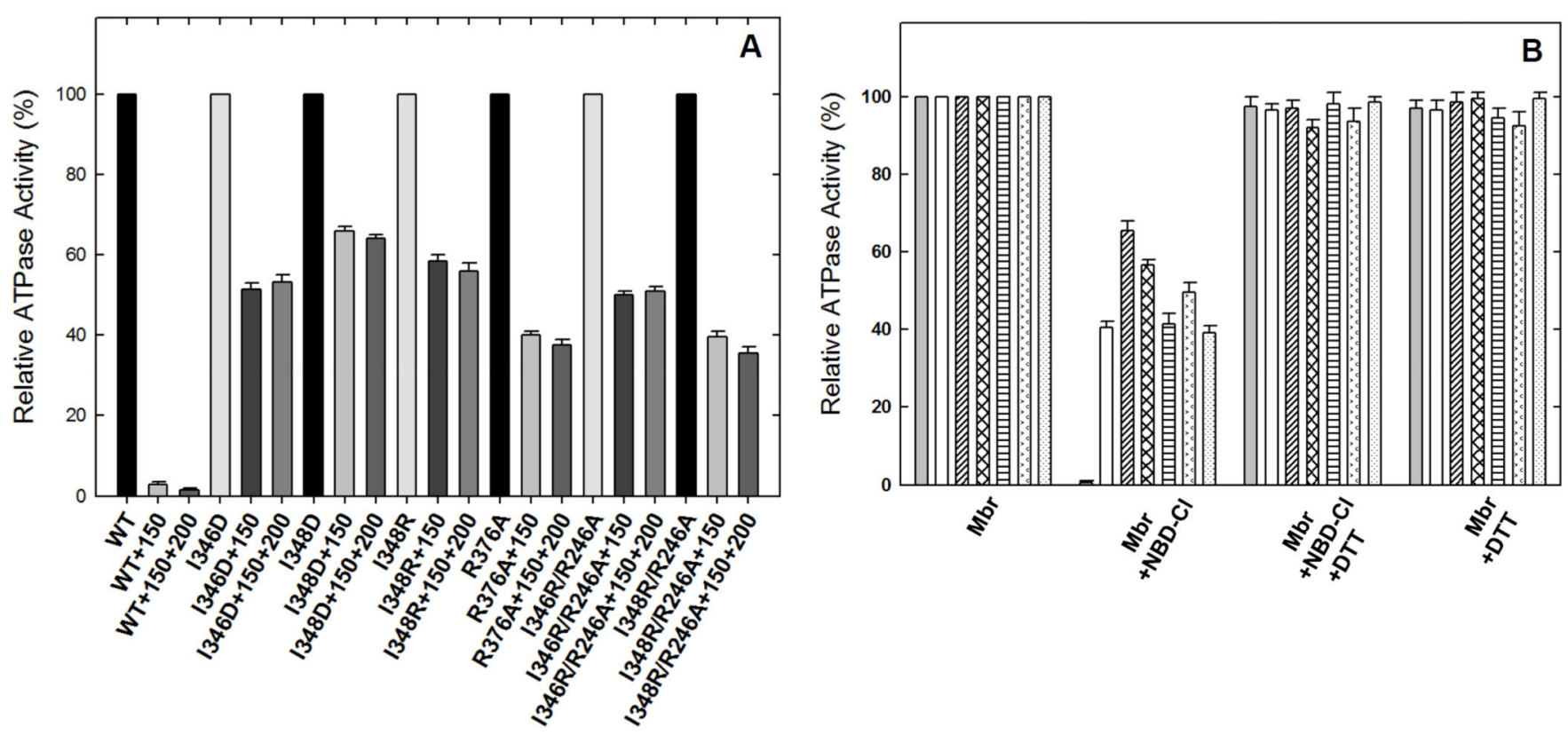

Figure 4. Effect of an extra pulse of NBD-Cl and reversal of NBD-Cl effect in the presence of DTT

A: Membrane bound $\mathrm{F}_{1} \mathrm{~F}_{\mathrm{o}}$ ATP synthase was inhibited with $150 \mu \mathrm{M}$ NBD-Cl for 60 min under conditions as described in Fig. 3. Then, an additional amount of $200 \mu \mathrm{M}$ NBD-Cl was added and incubation continued for $1 \mathrm{hr}$ before assay. $\mathrm{B}$ : Membrane bound $\mathrm{F}_{1} \mathrm{~F}_{\mathrm{o}}$ ATP synthase (Mbr) was incubated with or without $150 \mu \mathrm{M}$ NBD-Cl for 60 min under conditions as described in Fig. 3. The degree of inhibition was examined. In parallel samples, $4 \mathrm{mM}$ DTT was then added, and incubation continued for a further $60 \mathrm{~min}$ before assay. Each bar graph represents wild-type, aI346D, aI348D, aI348R, aR376A, aI346R/ßR246A, and aI348R/ $\beta$ R246A from left to right. For clarity, only mutants retaining residual activity after NBD-Cl inhibition are shown. 

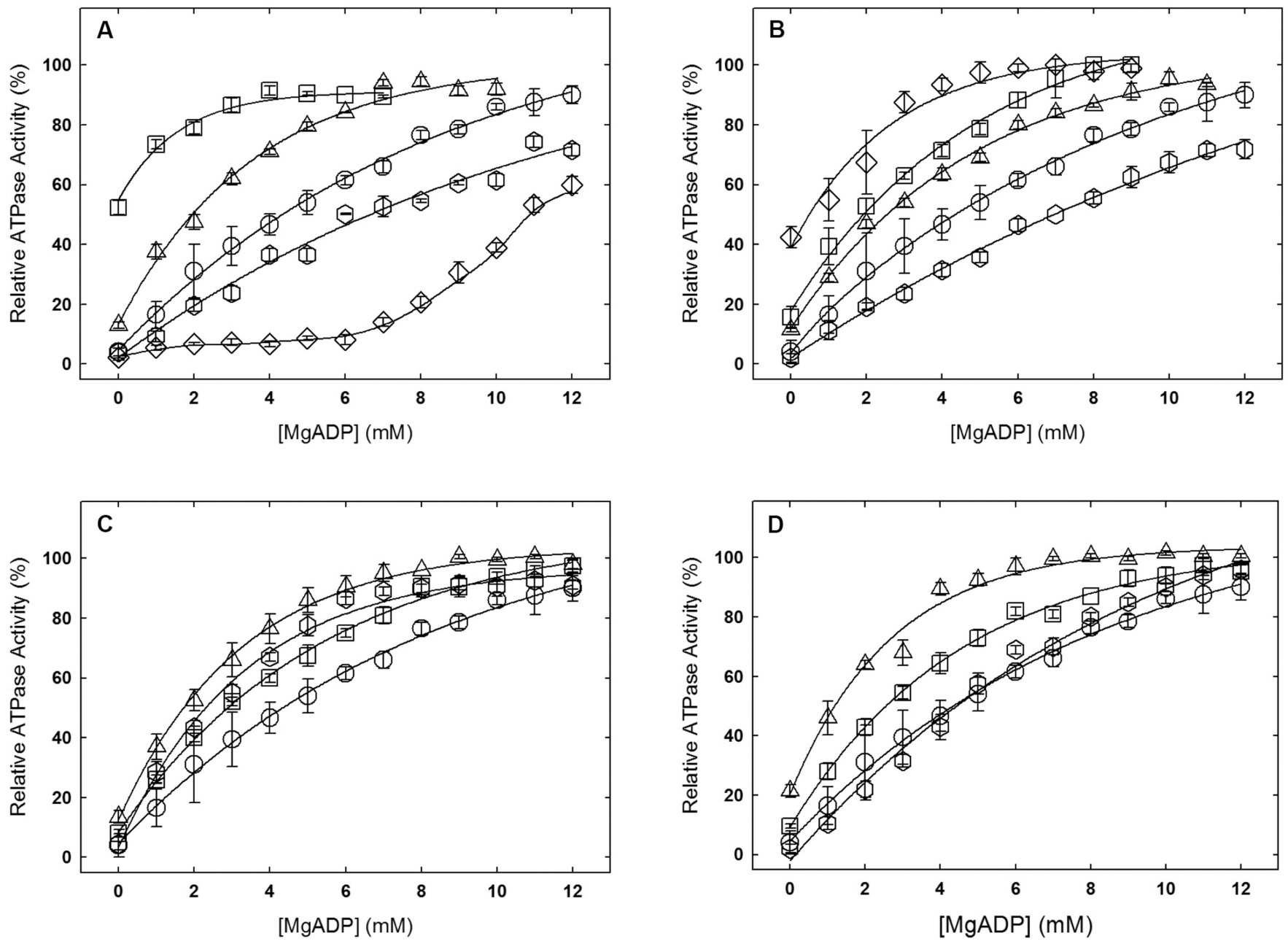

Figure 5. MgADP induced protection against NBD-Cl inhibition

Wild-type and mutant membrane bound $\mathrm{F}_{1} \mathrm{~F}_{\mathrm{o}}$ were preincubated for $1 \mathrm{~h}$ at room temperature with varied concentrations of MgADP, then $150 \mu \mathrm{M}$ NBD-Cl was added, and incubation continued at room temperature in the dark for 1 hour. Aliquots were assayed for ATPase activity. A: wild-type $(\bigcirc)$, a I346A $(\circlearrowright)$, a I346D ( $\square)$, a I346Q $(\Delta)$, and a I346R $(\diamond)$; B:

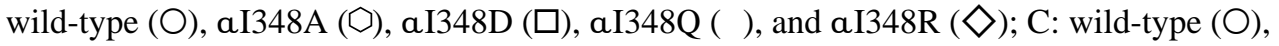

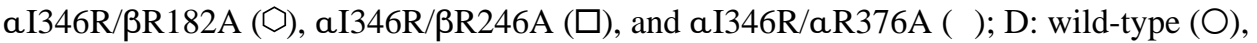

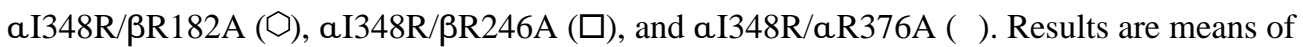
at least three independent experiments and agreed within $\pm 10 \%$. 

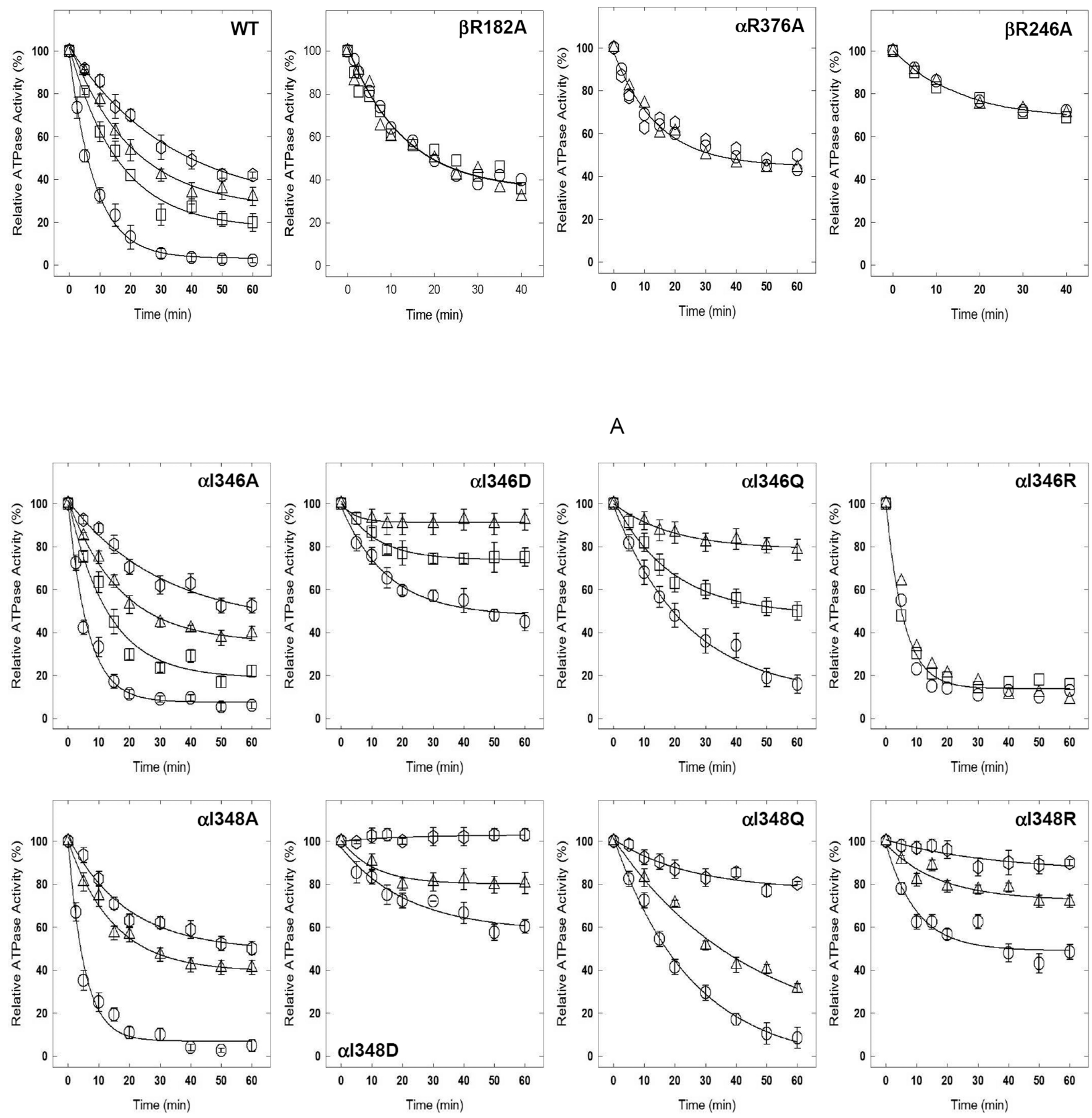

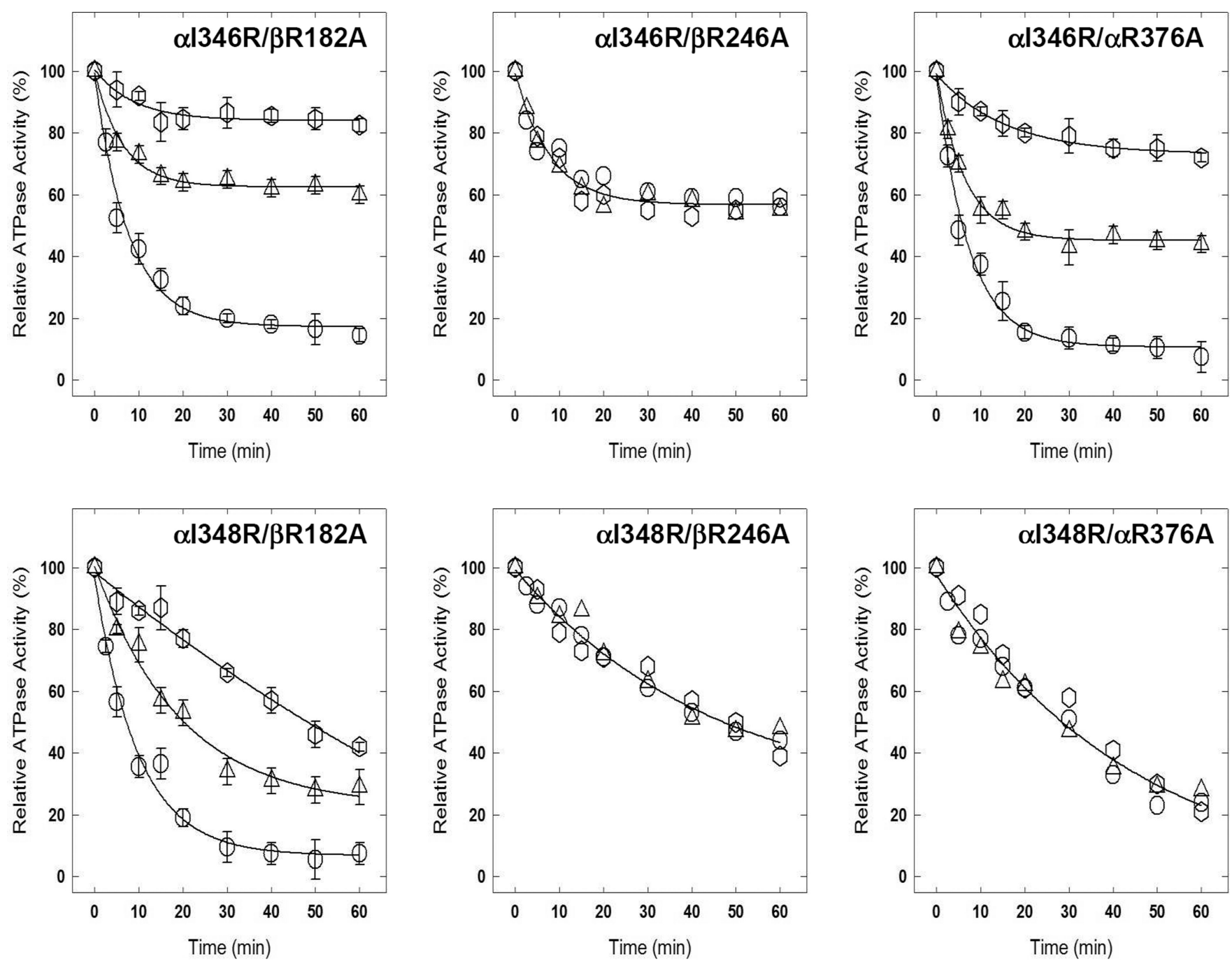

Figure 6. MgPi protection against NBD-Cl inhibition of membrane bound $\mathrm{F}_{1} \mathrm{~F}_{\mathbf{0}}$ ATPase activity $\mathrm{F}_{1} \mathrm{~F}_{\mathrm{o}}$ membranes were preincubated with $\mathrm{MgPi}$ at zero, $2.5,5$, or $10 \mathrm{mM}$ concentration as shown, for $60 \mathrm{~min}$ at room temperature. Then $150 \mu \mathrm{M} \mathrm{NBD}-\mathrm{Cl}$ was added and aliquots withdrawn for assay at time intervals as shown. Remaining ATPase activity was plotted against time of incubation with NBD-Cl. $\bigcirc$, no Pi added; $\square, 2.5 \mathrm{mM} \mathrm{Pi} ; \Delta, 5 \mathrm{mM} \mathrm{Pi} ; \bigcirc, 10$ $\mathrm{mM}$ Pi. Each data point represents the average of at least three individual experiments using 2-3 independent membrane preparations. Results agreed within $\pm 10 \%$. 

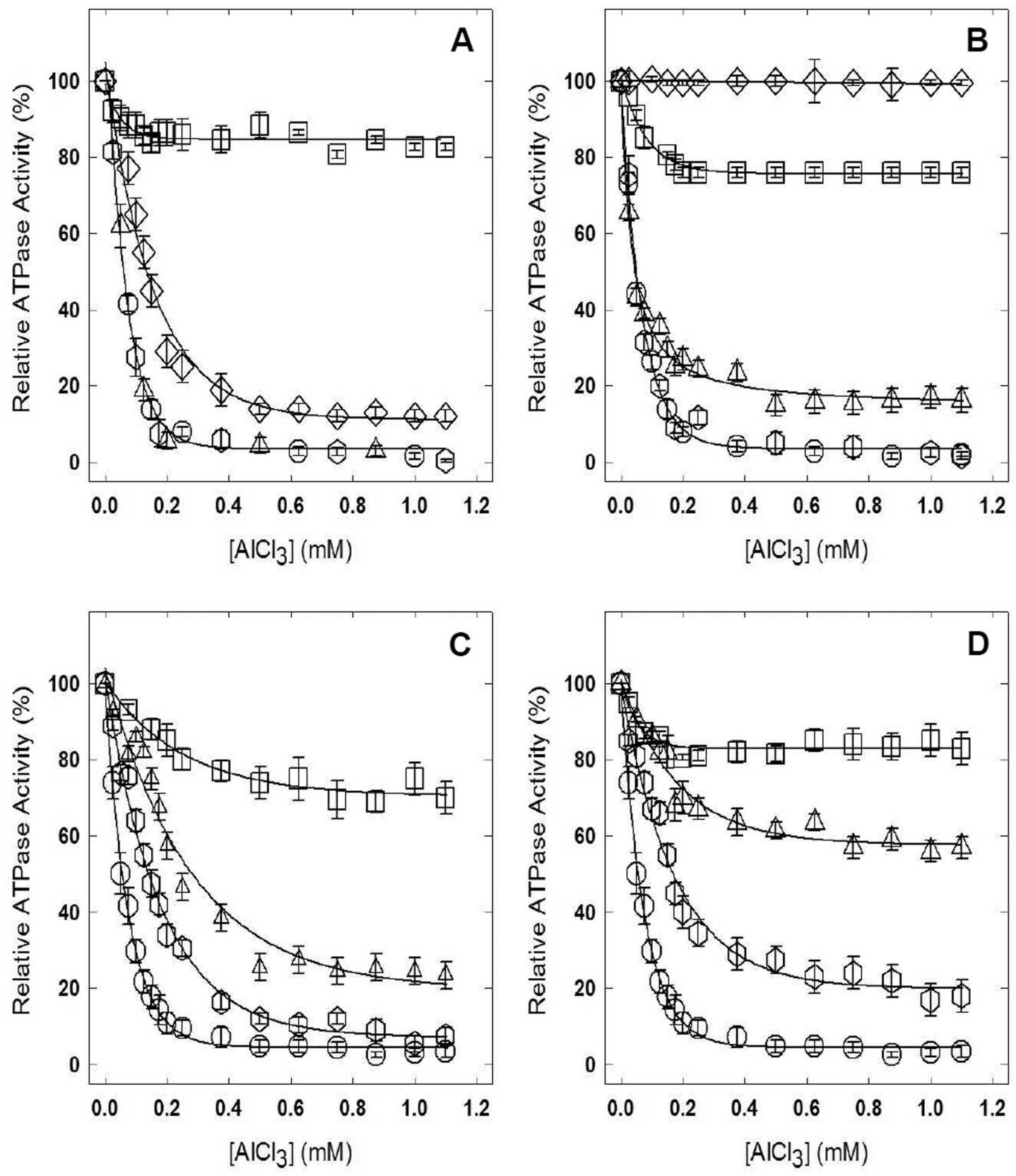

Arch Biochem Biophys. Author manuscript; available in PMC 2018 April 04. 

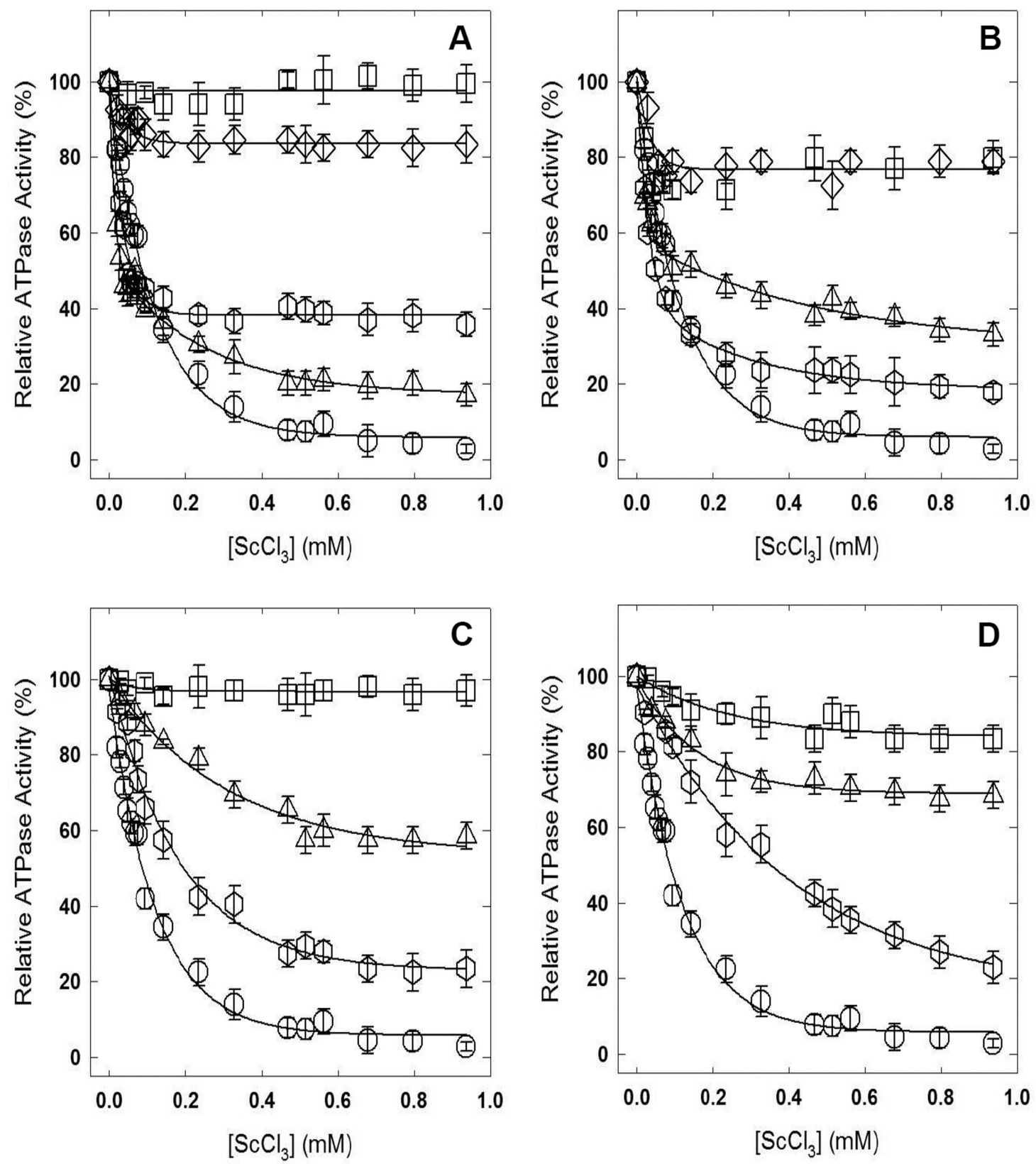

Figure 7. Fluoroaluminate and fluoroscandium induced inhibition of membrane bound $\mathbf{F}_{1} \mathbf{F}_{\mathbf{o}}$ ATPase activity

Membranes were preincubated for $60 \mathrm{~min}$ at room temperature with $1 \mathrm{mM} \mathrm{MgADP}, 10 \mathrm{mM}$ $\mathrm{NaF}$, and reported concentrations of $\mathrm{AlCl}_{3}(7 \mathrm{~A})$ or $\mathrm{ScCl}_{3}(7 \mathrm{~B})$. Then $1 \mathrm{ml}$ of ATP cocktail was added and ATPase activity determined. A: wild-type $(\bigcirc)$, aI346A $(\bigcirc)$, aI346D ( $\square$ ), aI346Q $(\Delta)$, and aI346R $(\diamond)$; B: wild-type $(O)$, aI348A (๑), aI348D ( $\square)$, aI348Q $(\Delta)$,

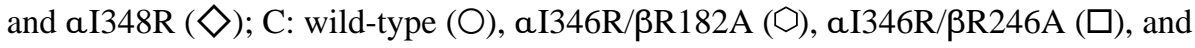

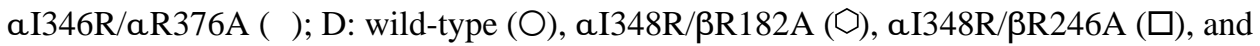
aI348R/aR376A $(\Delta)$. All the data points are means of at least three duplicate experiments using $2-3$ independent membrane preparations. Variation was $\pm 10 \%$ between different experiments. 

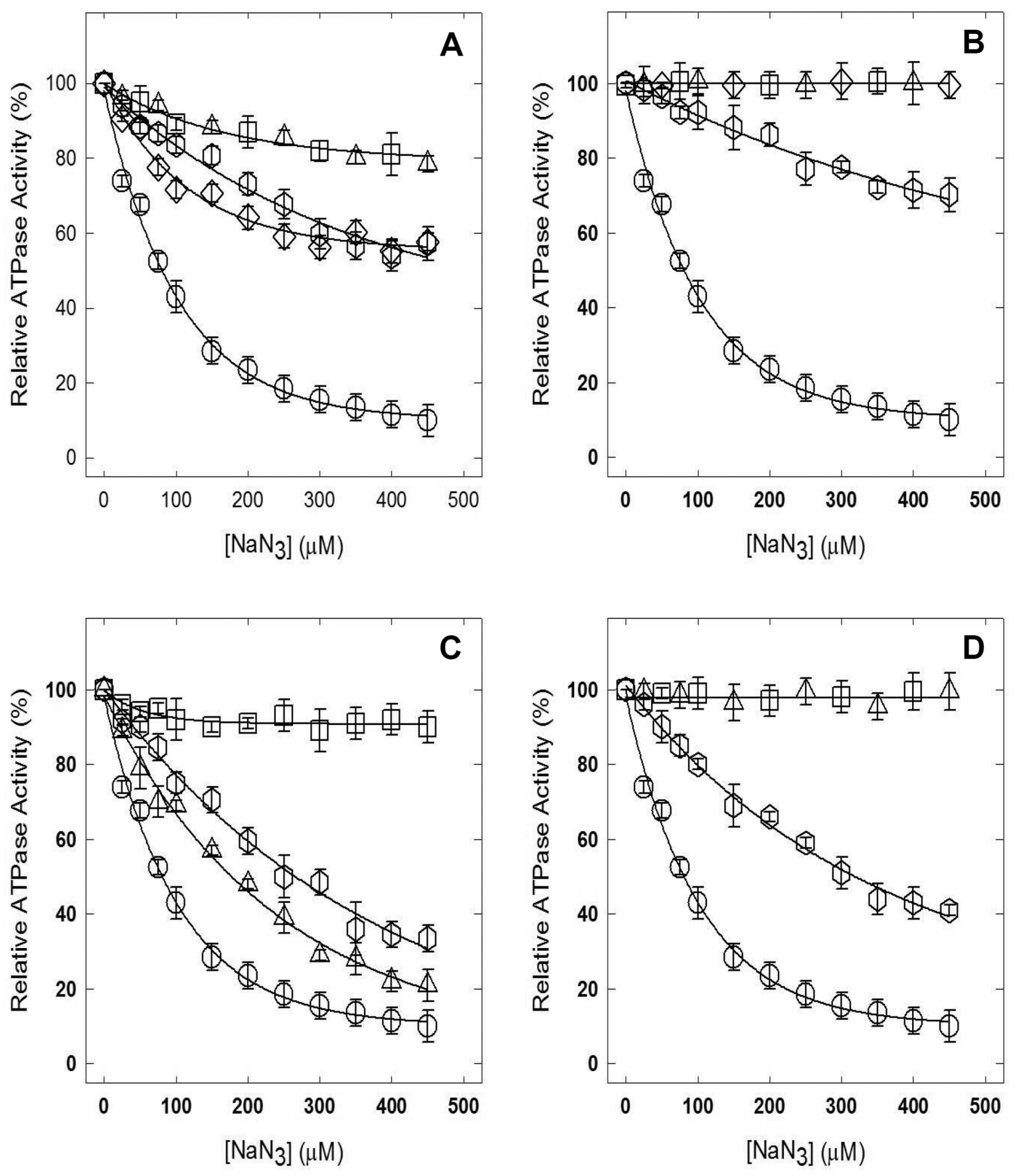

Figure 8. Sodium azide induced inhibition of membrane bound $F_{1} F_{0}$ ATPase activity Sodium azide was added directly to the membranes and incubated for $30 \mathrm{~min}$ before ATPase assay. More details are provided in materials and Methods. A: wild-type $(O)$, aI346A $(\triangle)$, aI346D ( $\square)$, aI346Q $(\Delta)$, and aI346R $(\diamond)$; B: wild-type $(\bigcirc)$, aI348A ( () ), aI348D ( $\square)$, aI348Q $(\Delta)$, and aI348R $(\diamond)$; C: wild-type $(\bigcirc), a I 346 R / \beta R 182 A(\bigcirc), a I 346 R / \beta R 246 A$

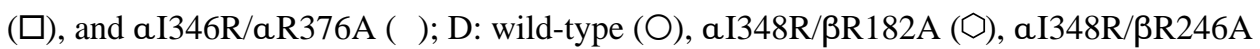

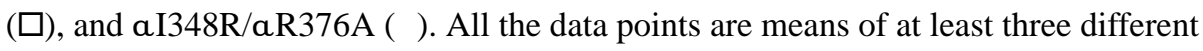


experiments using 2-3 independent membrane preparations. Variation was $\pm 10 \%$ between different experiments. 
TABLE 1

List of oligonucleotides

\begin{tabular}{|c|c|c|}
\hline Plasmids & Mutations & Sequence \\
\hline pZA65 & aI346A & CGACCAACGTGGCGTCCATTACCGATGG \\
\hline pZA66 & aI346D & CCGACCAACGTA $\underline{\text { GACTCCATTACCGATGG }}$ \\
\hline pZA67 & aI346Q & CCGACCAACGTACAGTCCATTACCGATGG \\
\hline pZA68 & aI346R & CGACCAACGTAAGATCTATTACCGATGG \\
\hline pZA69 & aI348A & CCAACGTAATCAGCGCTACCGATGGTCAG \\
\hline pZA70 & aI348D & CCAACGTAATCTC $\underline{\text { AGATACCGATGGTCAG }}$ \\
\hline pZA71 & aI348Q & CCAACGTAATCTCCCAGACCGATGGTCAG \\
\hline pZA72 & aI348R & CGTAATCTCWAGAACCGATGGTCAGATC \\
\hline pZA24 & $\beta \mathrm{R} 182 \mathrm{~A}^{a}$ & GGCGTAGGTGAAGCTACTCGTGAGGG \\
\hline pZA73 & aR376A & CGGGTATTTCCGTATCGGCCGTTGGTGGTGCAGC \\
\hline pZA7 & $\beta R 246 A^{b}$ & GACAACATCTATGCATACACCCTGGC \\
\hline pZA74 & aI346R/aR182A & pZA24 fragment added on plasmid pZA68 \\
\hline pZA75 & aI346R/aR376A & pZA73 fragment added on plasmid pZA68 \\
\hline pZA76 & $\alpha \mathrm{I} 346 \mathrm{R} / \beta \mathrm{R} 246 \mathrm{~A}$ & pZA768 fragment added on plasmid pZA7 \\
\hline pZA77 & aI348R/aR182A & pZA24 fragment added on plasmid pZA72 \\
\hline pZA78 & aI348R/aR376A & pZA73 fragment added on plasmid pZA72 \\
\hline pZA79 & $\alpha \mathrm{I} 348 \mathrm{R} / \beta \mathrm{R} 246 \mathrm{~A}$ & pZA72 fragment added on plasmid pZA7 \\
\hline
\end{tabular}

Bold and underline bases introduce the mutation and new restriction site.

taken from ref (9)

$b_{\text {taken from ref (15) }}$ 
TABLE 2

Effects of single and double a I346 and a I348 mutations on cell growth and ATPase activity

\begin{tabular}{llll}
\hline $\boldsymbol{a}_{\text {Mutation }}$ & $\begin{array}{l}\boldsymbol{b}_{\text {Growth }} \\
\text { On succinate }\end{array}$ & $\begin{array}{l}\text { Growth yield in } \\
\text { in limiting glucose } \\
(\boldsymbol{\%})\end{array}$ & $\begin{array}{l}\boldsymbol{c} \text { ATPase } \\
\text { Activity } \\
(\boldsymbol{\mu m o l} / \mathbf{m i n} / \boldsymbol{m} \boldsymbol{g})\end{array}$ \\
\hline Wild-type & ++++ & 100 & 28 \\
Null & - & 47 & 0 \\
a I346A & +++ & 90 & 6.9 \\
a I346D & ++ & 76 & 2.56 \\
a I346Q & ++ & 67 & 6.81 \\
a I346R & ++ & 74 & 3.74 \\
a I348A & +++ & 93 & 5.54 \\
a I348D & ++ & 78 & 0.08 \\
a I348Q & ++ & 66 & 4.54 \\
a I348R & + & 64 & 0.04 \\
a R182A & - & 47 & 0.07 \\
a R376A & - & 56 & 0.065 \\
$\beta$ R246A ${ }^{d}$ & - & 50 & 0.25 \\
a I346R/a R182A & +++ & 77 & 4.61 \\
a I346R/a R376A & +++ & 83 & 4.97 \\
a I346R/ $\beta$ R246A & + & 60 & 1.24 \\
a I348R/a R182A & +++ & 79 & 4.87 \\
a I348R/a R376A & + & 53 & 1.32 \\
a I348R/ $\beta$ R246A & + & 55 & 0.95 \\
\hline & & &
\end{tabular}

${ }^{a}$ Wild-type, pBWU13.4/DK8; Null, pUC118/DK8. All mutants were expressed with the $\beta Y 331 \mathrm{~W}$ mutation also present, which does not significantly affect growth. Data are means of four experiments each.

${ }^{b}$ Growth on succinate plates after 3 days estimated by eye. ++++, heavy growth; +++, substantial growth; ++, light growth; +,very light growth; -, no growth. Qualitative growth estimation on succinate plates is a widely used and more reliable method then the growth estimation on liquid succinate media as the rate of growth for wild-type and mutants could be different.

${ }^{c}$ ATPase activity measured at $37^{\circ} \mathrm{C}$ and expressed as $\mu \mathrm{mol}$ ATP hydrolyzed $/ \mathrm{min} / \mathrm{mg}$ of membrane protein. Individual experimental points are the mean of duplicate assay tubes. Data are derived from at least two separate membrane preparations. Results from separate membrane preparations were in excellent agreement within $\pm 10 \%$.

$d_{\text {Data taken from ref (9) }}$ 